\title{
In Vitro Evaluation of Fusiform-Shaped Stents for Wide-Neck Intracranial Aneurysm Treatment
}

\author{
Zhen Yu Jia, $\mathrm{MD}^{1,2^{\star}}$, Yuan Yuan Jiang, $\mathrm{MD}^{3^{*}}$, Jung Min Woo, $\mathrm{BS}^{1}$, Seon Moon Hwang, $\mathrm{BS}^{1}$,

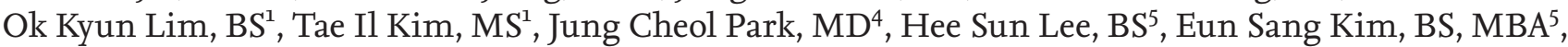 \\ Deok Hee Lee, MD, $\mathrm{PhD}^{1}$ \\ ${ }^{1}$ Departments of Radiology and Research Institute of Radiology, Asan Medical Center, University of Ulsan College of Medicine, Seoul, Korea \\ ${ }^{2}$ Department of Radiology, The First Affiliated Hospital of Nanjing Medical University, Nanjing, China \\ ${ }^{3}$ Department of Medical Biotechnology, Dongguk University, Goyang, Korea \\ ${ }^{4}$ Department of Neurosurgery, Asan Medical Center, University of Ulsan College of Medicine, Seoul, Korea \\ ${ }^{5}$ Angiovention, Goyang, Korea
}

Purpose: Wide-neck aneurysms (WNAs) associated with a dilated parent artery (PA) are not uncommon morphological abnormalities and usually cause inappropriate wall apposition and incomplete neck coverage of a tubular stent in stent-assisted coiling of aneurysms. We aimed to introduce a fusiform-shaped stent (FSS) and test its effectiveness in treating intracranial WNAs associated with a dilated PA using a three-dimensional (3D) model.

Materials and Methods: Two FSS types were designed with the middle one-third segment dilated by 10\% (FSS10) and 20\% (FSS20) and were compared with the tubular-shaped stent (TSS). A patient-specific 3D WNA model was prototyped and produced, and in vitro stent placement was performed. Angiographic images of the three stent types were analyzed and compared using predetermined parameters.

Results: The stent lumens were significantly larger in FSS10 and FSS20 than in TSS in the middle segments ( $P=0.046)$, particularly FSS20 ( $P=0.018)$. The non-covered area at the ostium tended to be smaller in FSS10 and FSS20 than in TSS, but the difference was not significant ( $P>0.05$ ). The stent length was significantly longer in FSS10 and FSS20 than in TSS. The stent cell size was significantly larger in FSS than in TSS.

Conclusion: Better vessel wall apposition and aneurysmal neck coverage was observed for FSS than for TSS. No significant difference was observed between FSS10 and FSS20.

Key Words: Intracranial aneurysm; Endovascular procedure; Stents; Self-expandable metal stent; Proof of concept experiment

\section{INTRODUCTION}

Endovascular aneurysm treatment has proven to have better outcomes than clipping for intracranial aneurysms, ${ }^{1-3}$ and with the advent of various self-expanding stents that served as scaffolds to prevent coils herniating into the par- ent artery (PA), wide-neck aneurysms (WNAs) have also become an indication for endovascular embolization. ${ }^{4-7}$ However, use of stent-assisted coiling with a tubular-designed stent for WNAs is sometimes difficult because of dilation of the vessel lumen of the PA, which may cause inappropriate abutting of a

\section{Correspondence to: Deok Hee Lee, MD, PhD Department of Radiology and Research Institute of Radiology, Asan Medical Center, University of Ulsan College of Medicine, 88 Olympic-ro 43-gil, Songpa-gu, Seoul 05505, Korea Tel: +82-2-3010-5944 \\ Fax: +82-2-476-0090 \\ E-mail:dhlee@amc.seoul.kr \\ Received: February 21, 2018 \\ Revised: May 30, 2018 \\ Accepted: June 29, 2018 \\ *These authors contributed equally to this work and should be considered co-first authors.}

Copyright $\odot 2018$ Korean Society of Interventional Neuroradiology

This is an Open Access article distributed under the terms of the Creative Commons Attribution Non-Commercial License (http://creativecommons.org/licenses/by-nc/3.0) which permits unrestricted non-commercial use, distribution, and reproduction in any medium, provided the original work is properly cited. 
stent to the vessel wall and incomplete coverage of the ostium. $^{8}$ Dilation of the PA had been reported as a not uncommon morphological abnormality in the distal internal carotid artery (ICA) with WNAs. ${ }^{9,10}$ Although the push-and-pull technique can make the middle part of a braid stent bulge to provide better mesh coverage at the ostium and better vessel wall apposition, the result is not satisfactory sometimes because of the limitations of the original design (Fig. 1A-C).

To overcome the limitations of currently available tubular-shaped stents (TSSs) in the treatment of WNAs with a dilated PA, we hypothesized that a self-expanding fusiform-shaped stent (FSS) designed with a bulging middle segment would achieve better wall apposition and better ostial coverage as well (Fig. 1D). The purpose of this in vitro study was to prove the concept of the FSS device for the endovascular treatment of WNAs associated with a dilated PA using a three-dimensional (3D) aneurysmal model.

\section{MATERIALS AND METHODS}

\section{Fabrication of TSS and FSS}

We fabricated three different prototype self-expanding stents by weaving a 0.0025-inch-diameter Nitinol wire (Furukawa Techno Material Co. Ltd., Kanagawa, Japan) (Fig. 1E). TSS was designed to have a body diameter of $4 \mathrm{~mm}$ and a length of $25 \mathrm{~mm}$. Both ends were flared with the maximum diameter of $6 \mathrm{~mm}$ at the margin. The two ends of the FSSs (each end one third length of the stent) were basically same with TSS, whereas the mid portion had a bulging shape (mid one-third segment). We made the FSSs with two different diameters of the bulging segment. FSS10 is an FSS with a bulging segment maximum diameter of $4.4 \mathrm{~mm}, 10 \%$ greater than the nominal body diameter. FFS20 has a bulging segment maximum diameter of $4.8 \mathrm{~mm}$ (20\% greater). Each type of stent was prepared as a pair to give six stents in total.

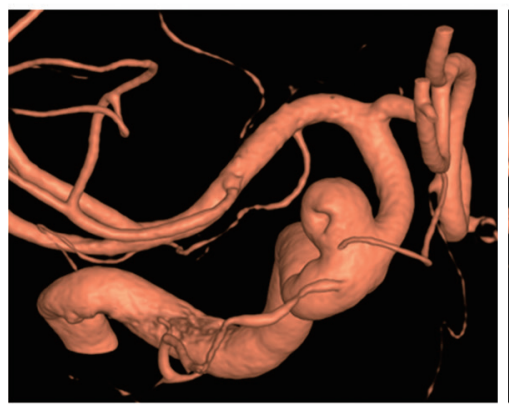

A
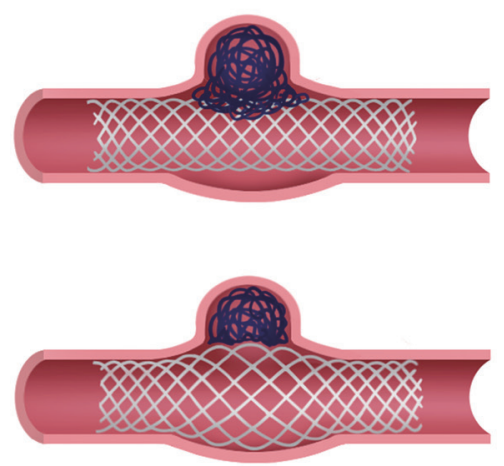
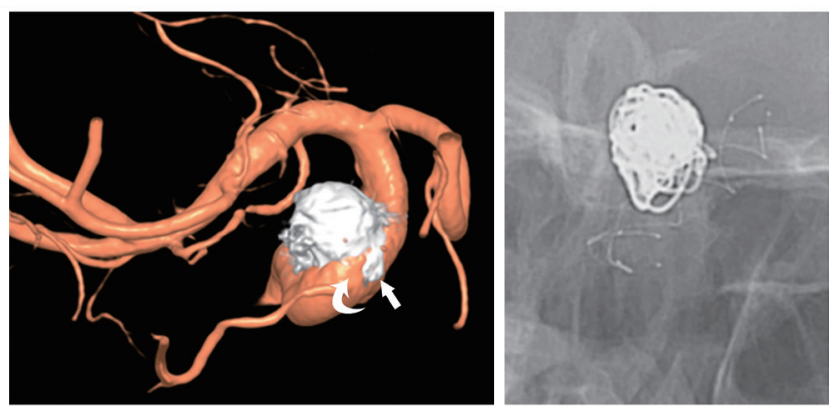

B
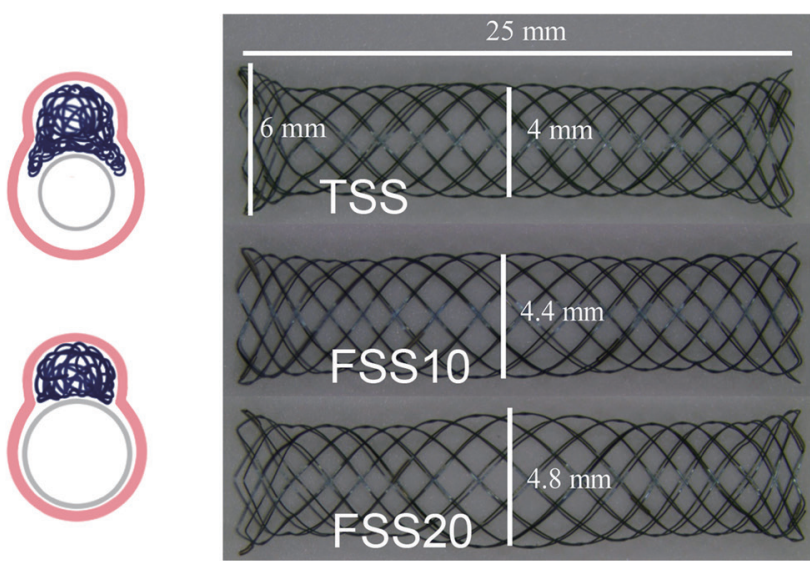

D
E

Fig. 1. Stent-assisted WNA coiling and concept design. (A-C) Right internal carotid artery (ICA) ophthalmic segment WNA with PA dilation (aneurysm neck diameter $5.3 \mathrm{~mm}$, proximal PA diameter $4.2 \mathrm{~mm}$, distal PA diameter $2.9 \mathrm{~mm}$, PA diameter at aneurysm neck $5.0 \mathrm{~mm}$ ) underwent stent-assisted coiling (LVIS blue, $4.5 \times 18 \mathrm{~mm}$ ). Final three-dimensional angiography showed several loops of coil protruding into the gap between the stent and vessel wall (arrow in B), and the irregular residual space between the stent, coils, and vessel wall (curved arrow in B, stent was visible on C). (D) Illustrations of a TSS and a FSS for coiling of WNAs with a dilated PA. The FSS shows better wall apposition and aneurysmal neck coverage. (E) Prototypes of the TSS and the FSS with middle segment dilated by 10\% and 20\%. WNA, wide-neck aneurysm; PA, parent artery; TSS, tubular-shaped stent; FSS, fusiform-shaped stent. 


\section{Silicone aneurysmal model}

A wide-neck distal ICA aneurysm associated with localized PA dilation was chosen (aneurysm volume of $535 \mathrm{~mm}^{3}$, ostium size of $8.5 \times 7.0 \mathrm{~mm}$, proximal PA diameter of $4.0 \mathrm{~mm}$, and distal PA diameter of $4.8 \mathrm{~mm}$ ). Simplified 3D volume data from rotational angiography was obtained from the volume-rendering image obtained on a Leonardo workstation (Siemens Healthcare, Erlangen, Germany) (Fig. 2A) and used to generate a rapid 3D printing prototype on a 3D printer (Project 3510 SD; 3D Systems, Rock Hill, SC, USA). Six silicone aneurysmal models were manufactured and mounted on a transparent acryl box full of silicone gel (Fig. 2B).

\section{Stent placement and angiography}

The silicone vascular model was connected by a closed circuit and infused with warm saline by using a peristaltic pump (Ecoline VC-280; Ismatec, Wertheim-Mondfeld, Germany). ${ }^{11}$ The tip of the 6 -Fr guiding catheter was placed right below the proximal stump of the silicone aneurysmal model. The stent was delivered through a 0.027-inch-sized microcatheter (Excelsior XT-27; Stryker Neurovascular, Fremont, CA, USA). A push-and-pull technique was used to place the stents under fluoroscopic guidance (Axiom Artis; Siemens Healthcare). The operator was blinded to the stent type during deployment of the stent to avoid bias caused by the delivery technique. The aneurysmal model was primed with 10\% saline-diluted iodine contrast media (Visipaque 270; GE Healthcare, Princeton, NJ, USA), and then volume-rendering and maximum intensity projection (MIP) images were obtained on the Leonardo workstation. For MIP, the contrast was adjusted so that the stent mesh could be differentiated from the vessel lumen and aneurysmal sac that were filled with diluted contrast media (Fig. 2C). Each of the six stents (three pairs) was placed in each of six silicone aneurysmal models; thus, 12 sets of data for each type of stent were acquired.

\section{Image analysis}

Imaging parameters were measured by using the vessel analysis tool in the Leonardo workstation and ImageJ $(\mathrm{NIH}$, Bethesda, MD, USA). We developed several parameters to investigate the efficacy of the stents. ANOVA was used to compare the repeated test results (Stata 12.0 for Mac [64bit Intel]). Bonferroni correction was performed for post hoc analyses.

\section{Centerline distance and stent expansion at the neck portion}

By using the vessel analysis tool of the Leonardo worksta-
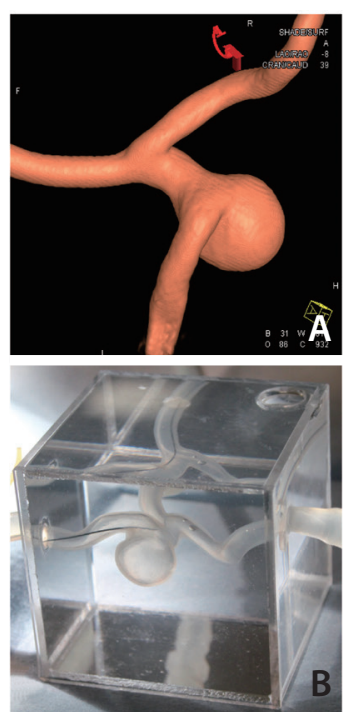
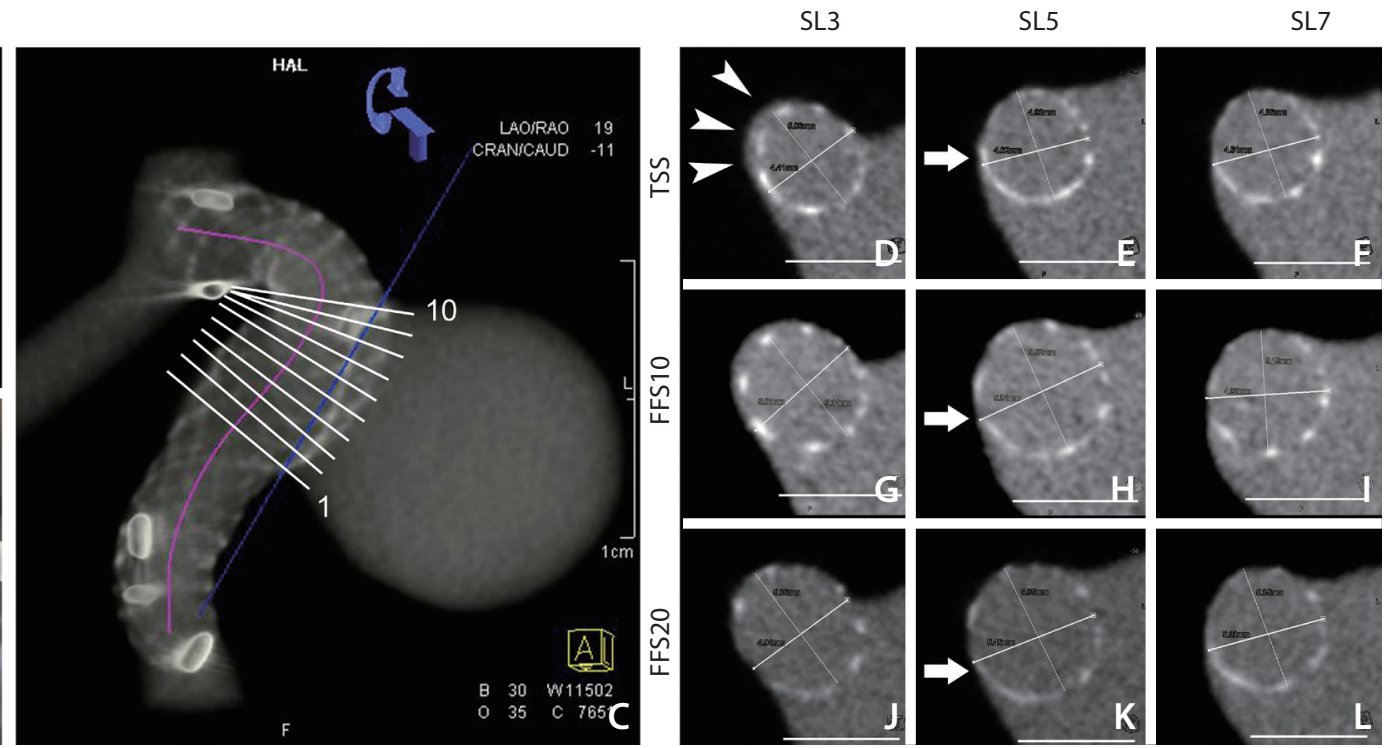

Fig. 2. Centerline distance and stent expansion. (A, B) Three-dimensional (3D) volume-rendering image of the aneurysm and the 3D prototype of silicone aneurysm model. (C) Reconstructed 3D image showing the placed stent in the silicone model on the Leonardo workstation. A centerline was drawn along the stented segment of parent artery to measure the length of the stent (purple line). The position of 10 different cross sections perpendicular to the centerline at the aneurysm neck portion was shown, which was used for stent lumen area measurement. (D-L) Representative images (slices 3, 5, and 7 marked in image $\mathbf{C}$ ) of each type of stent were compared and used for measuring the lumen area of the stents (cut-surface area). Note the gap between the TSS stent and the vessel wall (arrowheads in $\mathbf{D}$ ), and the better wall apposition evolution from the TSS to FSS20 (arrow in $\mathbf{E}, \mathbf{H}$, and $\mathbf{K})$. SL, slices; TSS, tubular-shaped stent; FSS, fusiform-shaped stent. 
tion, a centerline was drawn along the stented segment of the PA to measure the shortening or lengthening effect of the stents (Fig. 2C). We placed 10 different cross sections perpendicular to the centerline at the aneurysm ostium portion to measure the stent lumen area (cut-surface area) (Fig. 2C-L).

\section{Stent mesh coverage at the aneurysmal ostium and cell} area at the convex side

By placing a cutting plane at the neck that separated the aneurysmal sac from the PA, we could measure the stent-covered area and the non-covered area at the ostial portion (Fig. 3A-C). The cell area at the ostium portion was measured to see whether there was any stent deformity at the convex side. We chose the largest four-cell area at the profile view of the aneurysmal sac along the convex side of PA (Fig. 3D) because the larger stents gave better expansion.

\section{Stent wall apposition in the proximal and distal PA}

The proximal and distal PA segments covered by the stent mesh were chosen to obtain the cross-sectional images. Wall apposition to PA was measured by determining the ratio of the cross-sectional areas of the stent and PA lumen. The ratio was regarded as good when it was $>0.8$.
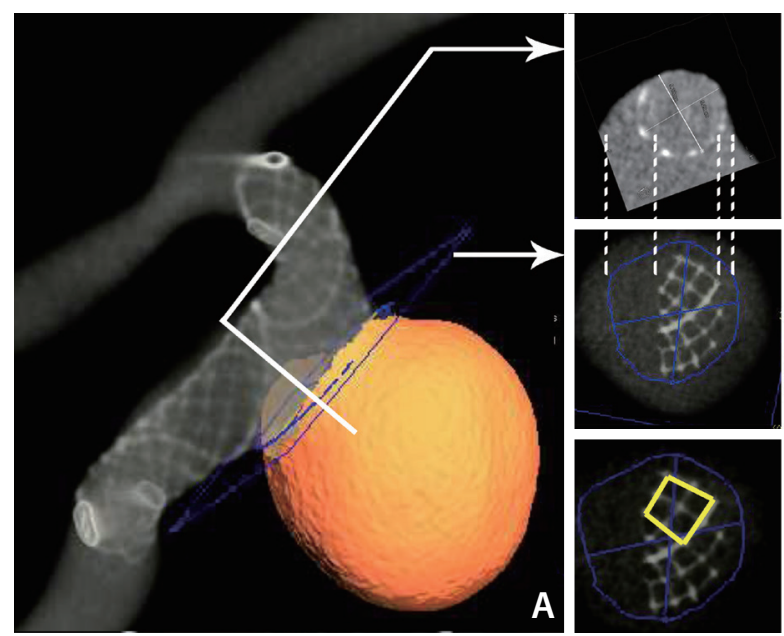

\section{C}

Fig. 3. Stent coverage at the aneurysm neck and cell area at the convex side. (A) Reconstructed 3D image showing the placed stent in the silicone model. (B) A cross section perpendicular to the centerline (corresponding to the white line shown in $\mathbf{A}$ ). (C) A section at the aneurysm neck to measure the neck coverage of the stent (the blue cutting plane shown in $\mathbf{A}$ ). The dashed lines between $(\mathbf{B})$ and $(\mathbf{C})$ showing the corresponding points in two planes. On image $(\mathbf{C})$, the stent-covered area and non-covered area of the aneurysm neck can be measured. (D) Measuring the four-cell size at the convex side of the stent.

\section{RESULTS}

\section{Centerline distance and stent expansion at the neck portion}

The centerline distance was significantly longer in both FSS10 and FSS20 than in TSS (P=0.006 and P=0.001, respectively). There was no significant difference between FSS10 and FSS20. In comparing the cut-surface areas of the stent lumen, significant differences were noted among the stents $(P=0.046)$. Post hoc analyses revealed that both FSS10 and FSS20 showed significantly larger cut-surface areas than those of TSS ( $P=0.024$ and $P=0.018$, respectively), but there was no significant difference between FSS10 and FSS20 (Fig. 4).

\section{Stent mesh coverage at the aneurysmal ostium and cell area at the convex side}

The non-covered area at the ostium tended to be smaller in FSS10 and FSS20 ( $P=0.327)$. The four-cell area at the convex side was significantly different among the stents $(P=0.002)$ and was significantly larger in both FSS10 and FSS20 than in TSS ( $P=0.018$ and $P=0.003$, respectively), but there was no difference between FSS10 and FSS20 (Fig. 4).

\section{Proximal and distal vessel wall apposition of the stents to the wall}

There was no significant difference in wall apposition among the stents in the proximal and distal segments of PA (both $P>0.05)$. However, when we compared the wall apposition ratio between the proximal and distal segments, we observed significantly better wall apposition in the proximal segment for all three types of stents $(P<0.05)$. The mean wall apposition ratios at the proximal and distal segments were $(0.86 \pm 0.04$ and $0.79 \pm 0.08$, respectively; $P<0.001)$. However, the wall apposition ratio at the distal segment was acceptable.

\section{DISCUSSION}

In the current study, we compared the efficacy of three types of self-expanding braided-type stents in the treatment of WNAs with significant PA dilation. Compared with TSS, both FSS10 and FSS20 showed better stent expansion, vessel wall apposition, and aneurysmal neck coverage as well as less stent shortening. 
The novel fusiform shape in the middle part of the tested stent design demonstrated its advantages mostly in two aspects. First, the good expansion of the middle part of the stent led to good apposition to the vessel wall at the segment of the aneurysmal neck, which may decrease delayed complications, such as stent thrombosis, caused by incomplete apposition of the stent. ${ }^{10}$ Though an oversized tubularshaped stent can achieve acceptable vessel wall apposition at the segment of the aneurysmal neck, the two ends will not get full expansion due to its larger diameter compared with the vessel lumen. Second, good expansion also resulted in better coverage of the ostium. The uncovered areas were relatively smaller in FSS10 and FSS20 than in TSS, which indicated that FSS can block protrusion of the coil back into the PA better than can TSS, and therefore, may avoid the irregular residual space between the stent and vessel wall and keep the inner surface of the lumen smooth (Fig. 1B). Apart from the simple braided stent, a fusiform stent may also be used in a flow-diverter to achieve a better wall apposition.

We noted that the currently available braided tubular design stents, such as the LVIS (MicroVention, Tustin, CA, USA), which is similar to the TSS in this study, may work to some extent similarly to our FSS. ${ }^{12,13}$ Intentional push-pull maneuvering during delivery of the stent may create a fusiform shape in the middle segment of the stent at the dilated PA. ${ }^{14}$ In this study, all three types of stents were deployed with the same push-and-pull technique by the same operator; however, a significant difference was still observed between TSS
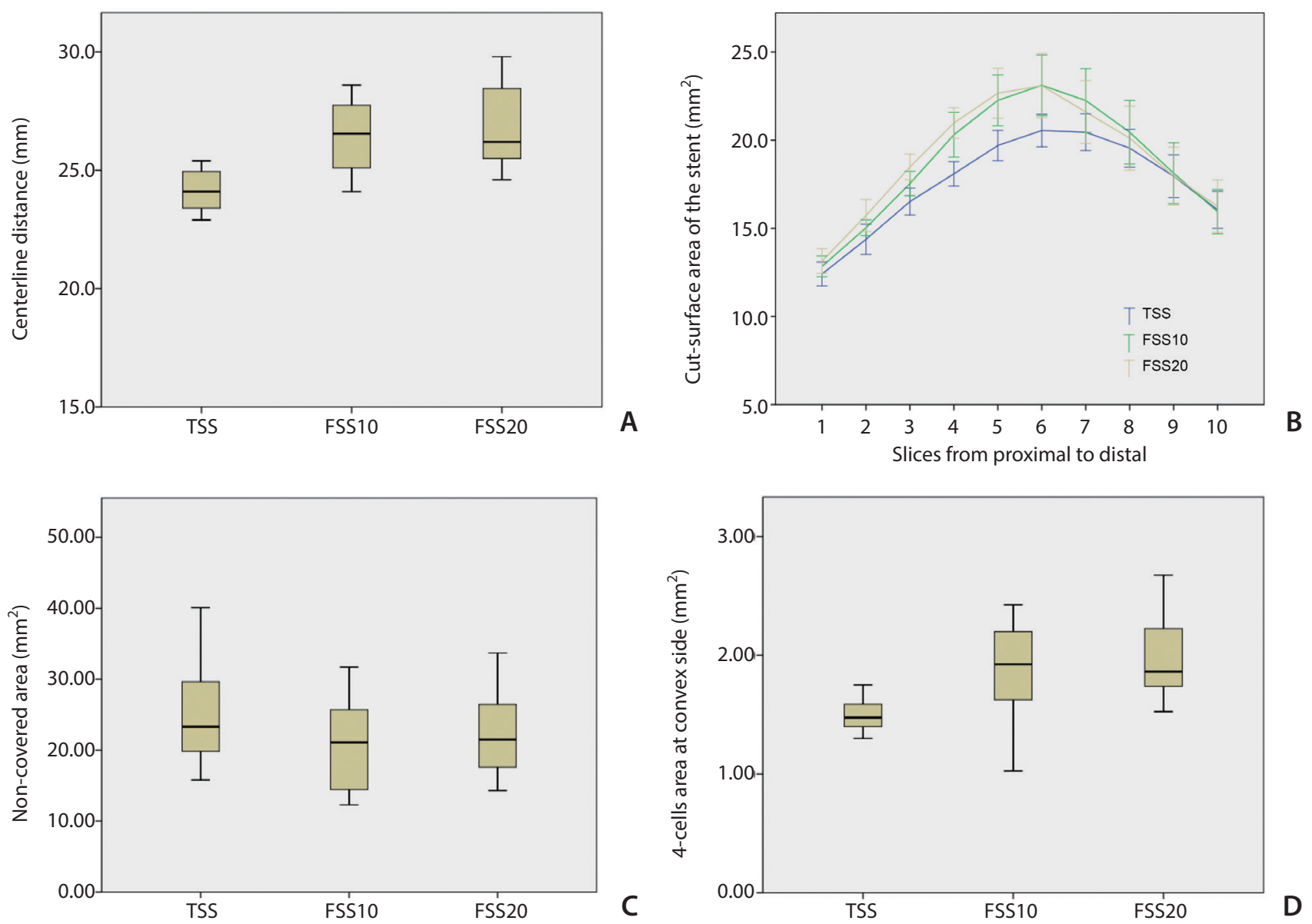

Fig. 4. Analytical results. (A) Comparison of the centerline distances of the stents showing that they are longer for FSS10 and FSS20 than for TSS. (B) Comparison of the stent lumen areas of the 10 sections along the stent among the three types of stents. FSS10 and FSS20 have significantly larger lumen areas than that of the TSS ( $P=0.024$ and $P=0.018$, respectively). (C) Comparison of the non-covered area sizes at the aneurysmal neck (measured in Fig. 3C) showing no significant difference. (D) Comparison of the four-cell areas at the convex side showing better expansion for FSS10 and FSS20. FSS, fusiform-shaped stent; FSS10, FSS with middle segment dilated by 10\%; FSS20, FSS with middle segment dilated by 20\%; TSS, tubular-shaped stent. 
and FSS10/FSS20 in the vessel apposition and aneurysmal neck coverage. Due to the initial fusiform design, the homogeneity of the mesh density would be better with a FSS than a TSS after deploying the stent with the push-and-pull technique. Compared with a TSS, a FSS does not need as much power to create the fusion form part, which can decrease the mechanical stress on the vessel wall and thus decrease the risk of intima injury. Additionally, the fusiform design of the stent decreases the shortening of the centerline distance relative to that of a TSS, which may facilitate accurate deployment of the stent.

Recently, the Barrel vascular remodeling device (Medtronic, Irvine, CA, USA), which is a self-expandable laser-cut stent developed with a bulged center section, was introduced for stent-assisted coil embolization of wide-necked bifurcation aneurysms. ${ }^{15}$ The stent was designed particularly for bifurcation aneurysms, and no experiences of use in side wall aneurysms with PA dilation have been reported yet. Considering the Barrel device for supporting the wide neck of a bifurcation aneurysm obviates use of multiple stents, we thought that this fusiform design might also be used for the same purpose.

The FSS and the TSS showed similar vessel wall apposition at both the proximal and distal portions of the stent. With both the FSS and the TSS, the proximal apposition was better than that of the distal portion, which may be attributed to the case-specific vessel geometry and push-and-pull technique. When we began to deploy the stent, there was not too much friction between the stent and vessel wall, so the wall apposition of the stent at the distal portion depended on the self-expansion of the stent. While at the end of deployment of the stent, the stent is immobilized by the vessel and the push-and-pull action can lead to better wall apposition. Future studies will be conducted to test if case-specific geometry prototypes will affect the apposition.

In the current study, we presented a fast 3D prototyping method for a case-specific vessel with radiographic volume information that has greater efficiency than that of an animal model for evaluation of device morphological designs and can be used to preliminarily study the efficacy of a stent before an in vivo study. The parameters applied to evaluate the efficacy of the stent can be correlated with clinical outcomes in the future and used to evaluate clinical prognosis. However, in vivo animal experiments are still necessary for making quantitative guides for choosing the size of the fusiform stent.
There were several limitations of this in vitro experiment that should be considered. First, only one wide-necked distal ICA aneurysm model was used. Because a variety of factors may affect wall apposition of a stent, such as vessel tortuosity and lumen discrepancy proximally and distally to the aneurysm, the stents should be tested in more models with different configurations. Second, our results were limited to braided stents, so we cannot generalize the results to laser-cut stents, since they tend to behave differently in the same PA morphology. Third, the silicone aneurysmal model cannot simulate all features of a human artery, so before human trials, animal experiments are necessary to test its biocompatibility.

\section{CONCLUSION}

Our results showed that the FSS gave better neck coverage than that of the TSS in the treatment of intracranial widenecked aneurysms through enhanced wall apposition, especially at the dilated segment of the PA without significant lengthening of the stent. There was little difference in the performance between FSS10 and FSS20. Further experiments should be carried out in more cases with various vascular geometries before clinical application.

\section{Acknowledgments}

This research was supported by a grant (HO13C0006) from Osong Innovation Center funded by the Ministry of Health \& Welfare, the Republic of Korea.

\section{REFERENCES}

1. Molyneux AJ, Kerr RS, Yu LM, Clarke M, Sneade M, Yarnold JA, et al. International subarachnoid aneurysm trial (ISAT) of neurosurgical clipping versus endovascular coiling in 2143 patients with ruptured intracranial aneurysms: a randomised comparison of effects on survival, dependency, seizures, rebleeding, subgroups, and aneurysm occlusion. Lancet 2005;366:809-817

2. Jeong HW, Seo JH, Kim ST, Jung CK, Suh SI. Clinical practice guideline for the management of intracranial aneurysms. Neurointervention 2014;9:63-71

3. Yu M, Liu F, Jiang S, Nie B. Stent-assisted coiling for the treatment of ruptured micro-intracranial wide-necked aneurysms. 
Interv Neuroradiol 2015;21:40-43

4. Fiorella D, Albuquerque FC, Han P, McDougall CG. Preliminary experience using the Neuroform stent for the treatment of cerebral aneurysms. Neurosurgery 2004;54:6-16; discussion 16-17

5. Benitez RP, Silva MT, Klem J, Veznedaroglu E, Rosenwasser RH. Endovascular occlusion of wide-necked aneurysms with a new intracranial microstent (Neuroform) and detachable coils. Neurosurgery 2004;54:1359-1367; discussion 1368

6. Krischek O, Miloslavski E, Fischer S, Shrivastava S, Henkes H. A comparison of functional and physical properties of self-expanding intracranial stents [Neuroform3, Wingspan, Solitaire, Leo+, Enterprise]. Minim Invasive Neurosurg 2011;54:21-28

7. Cho SH, Jo WI, Jo YE, Yang KH, Park JC, Lee DH. Bench-top comparison of physical properties of 4 commercially-available self-expanding intracranial stents. Neurointervention 2017;12:3139

8. Heller RS, Miele WR, Do-Dai DD, Malek AM. Crescent sign on magnetic resonance angiography revealing incomplete stent apposition: correlation with diffusion-weighted changes in stent-mediated coil embolization of aneurysms. J Neurosurg 2011;115:624-632

9. Lee JW, Woo JM, Lim OK, Jo Y, Kim JK, Kim ES, et al. Enlarged parent artery lumen at aneurysmal-neck segment in wide- necked distal internal carotid artery aneurysms. Neurointervention 2015;10:82-88

10. De Bock S, lannaccone F, De Santis G, De Beule M, Mortier P, Verhegghe $B$, et al. Our capricious vessels: the influence of stent design and vessel geometry on the mechanics of intracranial aneurysm stent deployment. J Biomech 2012;45:1353-1359

11. Lee DH, Hwang SM, Lim OK, Kim JK. In vitro observation of air bubbles during delivery of various detachable aneurysm embolization coils. Korean J Radio/ 2012;13:412-416

12. Herweh C, Nagel S, Pfaff J, Ulfert C, Wolf M, Bendszus M, et al. First experiences with the new Enterprise ${ }^{\circledR}$ Stent. Clin Neuroradio/ 2018;28:201-207

13. Zhang X, Zhong J, Gao H, Xu F, Bambakidis NC. Endovascular treatment of intracranial aneurysms with the LVIS device: a systematic review. J Neurointerv Surg 2017;9:553-557

14. Darflinger RJ, Chao K. Using the Barrel technique with the LVIS Jr (low-profile visualized intraluminal support) stent to treat a wide neck MCA bifurcation aneurysm. J Vasc Interv Neurol 2015;8:25-27

15. Muhl-Benninghaus R, Simgen A, Reith W, Yilmaz U. The Barrel stent: new treatment option for stent-assisted coiling of widenecked bifurcation aneurysms-results of a single-center study. J Neurointerv Surg 2017;9:1219-1222 